\title{
Selection of Donors for Living Donor Liver Transplantation
}

\author{
James F. Trotter
}

Key Points

1. The donor evaluation is performed in three staged phases to disqualify inappropriate candidates as early as possible in the process.

2. Approximately half the potential transplant recipients are unable to identify a suitable donor for evaluation.

3. Approximately half the individuals evaluated for living donation are found suitable.

4. The donor pool in many parts of the country is significantly limited by the high prevalence of obesity. (Liver Transpl 2003;9:S2-S7.)

$\mathrm{T}$ he selection of donors for adult-to-adult right hepatic lobe living donor liver transplantation (LDLT) is one of the most important aspects of this procedure. ${ }^{1-5}$ Criteria for donor selection are evolving as experience with the procedure increases. However, the two fundamental purposes of the donor evaluation are to ensure that (1) the donor operation may be performed safely, and (2) the donor is able to yield a suitable graft for the recipient. Donor safety is the single most important clinical issue related to LDLT. Concern over donor safely may be one of the reasons the number of LDLTs performed in the United States has declined since the widely publicized donor death that occurred January 2002. After this tragic event, many transplant surgeons and physicians have taken a more cautious approach to LDLT. As a result, the number of LDLT procedures decreased 31\% from 518 in 2001 to 359 in $2002 .{ }^{6}$ The current rate of LDLTs performed in 2003 is even lower, with a projected total number of cases (272 LDLTs by year's end) nearly $50 \%$ less than in 2001. This report gives a brief overview of the donor evaluation process, followed by discussion of current relevant topics in donor evaluation. CO.

From the University of Colorado Health Sciences Center, Denver,

Address reprint requests to James $F$. Trotter, $M D$, University of Colorado Health Sciences Center, 4200 E 9th Ave, B-154, Denver, CO 80209. Telephone: 303-372-8866; FAX: 303-372-8868; E-mail: James.Trotter@uchsc.edu

Copyright () 2003 by the American Association for the Study of Liver Diseases

1527-6465/03/0910-2002\$30.00/0

doi:10.1053/jlts.2003.50221

\section{Donor Selection}

After a patient with chronic liver disease is identified as a suitable candidate for LDLT, he or she may accept volunteers for donor evaluation. After the donor has contacted the transplant center, evaluation of the potential donor proceeds in a stepwise fashion so that unsuitable donors may be excluded as early as possible in the evaluation process.

Table 1 lists the three specific phases of donor evaluation used at our center. Phase 1 is designed to determine that the potential donor meets all the appropriate inclusion criteria for donation: appropriate blood type, age, body size, and relationship to the recipient. At our center, this initial phase of donor evaluation is performed by an experienced registered nurse over the telephone. The potential donor must be between the ages of 18 and 55 years, show a significant long-term relationship with the recipient, and have an identical or compatible blood type and no significant medical problems. No other immunologic matching other than blood type is required of the potential donor. In addition, height, weight, and a brief medical, psychosocial, and surgical history is documented, along with current medications. Serum electrolyte levels, blood count with differential, liver function tests, blood type, and hepatitis serological tests may be performed if the donor has no obvious contraindications to donation. Relative contraindications for donor evaluation discovered frequently in the early phase of donor evaluation include hypertension, hypercholesterolemia, previous significant abdominal surgery, and obesity. These issues must be considered in each potential donor case by case, based on the experience and judgment of the transplant team. In all cases, the safety and health of the donor are the primary considerations.

Approximately $50 \%$ of patients who are eligible for LDLT are unable to identify a potential donor who fulfills the donor criteria listed in Table $1 .{ }^{7}$ Reasons a patient is unable to identify an potential donor for evaluation often are complex and include estrangement from family/friends, no family/friends between the ages of 18 and 55 years, or a potential donor with an obvious medical problem (morbid obesity, active substance abuse, or significant obvious medical problem). One basic criterion at almost all centers is that the donor and recipient must have a significant long-term relation- 
Table 1. Phases of Living Donor Evaluation

Phase 1

Age $\geq 18$ and $\leq 55$ years

Identical or compatible blood type with recipient

Absence of previous significant abdominal surgery and/or medical problems

Significant long-term relationship with recipient

Normal liver function test results, serum electrolyte levels, complete blood count with differential cell count, and negative hepatitis B surface antigen and

Phase 2 hepatitis $\mathrm{C}$ antibody results

Complete medical history and physical examination

Laboratory tests: serum ferritin, iron, transferrin, ceruloplasmin, $\alpha_{1}$-antitrypsin level and phenotype, rapid plasmin reagin, cytomegalovirus antibody (immunoglobulin G), Epstein-Barr virus antibody (immunoglobulin G), antinuclear antibody, human immunodeficiency antibody, toxicology/substance abuse screen, urinalysis, and blood oxygen saturation Chest radiograph

Electrocardiogram

Formal surgical evaluation of donor

Anesthesia preoperative evaluation

Magnetic resonance imaging of the liver, biliary system, and hepatic vasculature

Phase 3

Other tests or consultations to clarify any potential problems uncovered during evaluation: e.g., endoscopic retrograde cholangiopancreatography, hepatic angiogram, liver biopsy, echocardiogram, and stress echocardiogram (some centers routinely perform these tests as part of the donor evaluation)

Data from Trotter et al. ${ }^{1}$

ship. Evaluation of nondirected donation (Good Samaritan) is not considered at most centers. Rarely, recipients eligible for LDLT refuse to accept LDLT as a treatment option because of safety concerns related to the donor's surgery. In our experience, less than $5 \%$ of patients decline LDLT for this reason. ${ }^{7}$

Body size compatibility between the donor and recipient is an important preliminary consideration in the donor evaluation. At our center, we require that the donor and recipient are within approximately 30\% body weight of each other. In most cases in which the recipient's body weight is $30 \%$ or greater of the donor's body weight, the donor will be unable to yield a large enough graft for the recipient. Other centers use specific formulas to assist in the prediction of whether the donor has adequate hepatic mass. Two formulas are used to assess graft size adequacy: (1) graft-recipient body weight ratio and (2) graft weight as a percentage of standard liver mass. ${ }^{8}$ Both are equally useful, with lower limits of graft acceptability of $0.8 \%$ to $1.0 \%$ for graftrecipient body weight ratio and $40 \%$ to $50 \%$ for percentage of standard liver mass. However, there may be a wider range of acceptability depending on the recipient's Child's class. ${ }^{9}$ For physically large recipients greater than $100 \mathrm{~kg}$, the likelihood of finding a donor physically large enough to yield a sufficiently large graft is small. At our center, the largest living donor liver transplant recipient weighed $104 \mathrm{~kg}$ at the time of surgery. As a result, LDLT has limited applicability in large patients because of the inability to identify a suitable donor.

After phase 1 data are obtained, the potential donor is presented to the transplant selection committee to decide whether to proceed with phase 2 (comprehensive donor evaluation). Phase 2 consists of additional testing, including a complete medical history and physical, psychosocial, surgical, and preoperative anesthesia evaluations. These tests must be performed at the transplant center where the donor surgery is to occur. To prevent an obvious conflict of interest, medical history and physical examination should be performed by a physician who is not attending the recipient. Donor psychosocial evaluation, an important component of the overall evaluation process, is discussed in another article. At our center, we have noted that almost all our donors are patients who, based on their excellent health, have little or no experience with medical procedures. As a result, we have a nurse meet with each potential donor to teach him or her about the critical logistics of the procedure (e.g., preanesthesia care, immediate postoperative care, and explanation and purpose of surgical drains and nasogastric tube). We also give each potential donor the opportunity to meet personally with another donor who has undergone the donor surgery.

Phase 3 of the evaluation is performed to follow up on any abnormalities discovered during phase 2 . At our center, approximately $60 \%$ of potential donors evaluated are found medically suitable and undergo donation. $^{7}$

There are important considerations that the transplant team must consider regarding the final decision of the suitability of a donor for donor surgery. Some potential donors decide not to donate based on information that they gain during the donor evaluation. In almost all cases, it is in the best interest of the donor not to reveal this decision directly to the recipient. It also is important for the donor to be given sufficient time to process all the information gained during the evaluation process so that he or she can make an informed and considered decision regarding the choice for donation. In many clinical settings, pressure on the transplant 
team and donor to make a final decision regarding donation is intense because of the severity of illness of the recipient. However, the integrity of the evaluation process must be maintained above all other interests, and the donor must be given sufficient time to make a careful decision regarding donation without external pressures.

After the donor has been approved for donor surgery, timing of the transplantation must be determined based on the medical condition of the recipient. Each recipient is maintained on the cadaveric transplant list up to the time of LDLT. In almost all cases, a cadaveric transplant is preferable to LDLT; therefore, the recipient would be eligible to receive a cadaveric donor up to the time of LDLT.

\section{Special Considerations and/or Issues in Donor Evaluation}

\section{Obesity}

Obesity (body mass index $[\mathbf{B M I}]>28 \mathrm{~kg} / \mathrm{m}^{2}$ ) in potential donors is an increasingly common problem because the prevalence of obesity is increasing in the United States. Currently, 21\% of the US population is obese. ${ }^{10}$ In general, obese individuals are unsuitable for donation. A recent study showed that $78 \%$ of potential donors with a BMI greater than $28 \mathrm{~kg} / \mathrm{m}^{2}$ had hepatic steatosis (>10\% steatosis) on liver biopsy. ${ }^{11}$ Most centers exclude donors who have greater than $10 \%$ steatosis on liver biopsy because in cadaveric transplantation, hepatic steatosis is associated with poor posttransplantation graft function. However, there are no data regarding outcomes after LDLT with the use of steatotic grafts. As a result, most centers exclude obese donors from evaluation. However, one report showed a poor correlation with BMI and hepatic steatosis. Ryan et $\mathrm{al}^{12}$ found that $73 \%$ of overweight (BMI $>25$ $\mathrm{kg} / \mathrm{m}^{2}$ ) donors had little or no hepatic fat and $9 \%$ of candidates with a BMI of $25 \mathrm{~kg} / \mathrm{m}^{2}$ or less had $10 \%$ or greater steatosis.

Another reason that obesity is a relative exclusion for donor evaluation relates to the increased risk for complications with the donor surgery. Studies have shown that risk for bleeding and postoperative complications is greater after hepatectomy in obese compared with nonobese patients. In addition, obese patients are more likely to have comorbid conditions (hypertension, hypercholesterolemia, and diabetes), which could increase the risk for postoperative complications after donor hepatectomy. ${ }^{13}$

Although an obese donor rarely is able to donate, selected overweight patients may be considered for living liver donation after careful evaluation. Distribution of fat in the body may be an important factor in determining suitability for donation. Patients with a greater waist-hip ratio have more abdominal fat and a greater incidence of hepatic steatosis. ${ }^{14}$ The increased distribution of abdominal fat also may increase the likelihood of abdominal wound complications after donor surgery. A greater waist-hip ratio is more common in men than women. Consequently, overweight nonobese women may be more suitable for donation than similar-sized men.

Regional variations in the prevalence of obesity can be important determinants in the suitability of the donor pool at transplant centers. The greatest regional prevalence of obesity is in the southeast and north central United States. ${ }^{10}$ The potential donor pool for LDLT centers in these regions may have significant difficulty in the identification of suitable donors because of the high incidence of obesity. However, the likelihood of identifying a nonobese donor for LDLT may be greater in the Rocky Mountain West, which has the lowest prevalence of obesity of any region in the United States.

\section{Donor Advocate}

An important recent development in the donor evaluation process is the recommendation for a donor advocate. The role of the donor advocate was created in large part as a result of the re-examination of the donor evaluation process after a well-publicized donor death in 2002. Currently, the Ad Hoc United Network for Organ Sharing (UNOS) Committee on Living Donor Liver Transplantation, ${ }^{15}$ the Secretary of Health and Human Services Advisory Committee of Organ Transplantation, ${ }^{16}$ and the New York State Health Board ${ }^{17}$ recommend that each LDLT center provide a donor advocate for each person evaluated for living liver transplant donation. The specific qualifications of the donor advocate have been left undefined by design. However, a donor advocate must be an employee of the medical center and have some ties to the transplant team to ensure they have a basic understanding of the transplantation process. The donor advocate could be a social worker, psychiatrist, or physician.

The primary role of the donor advocate is to "represent and advise the donor so as to ensure that the [appropriate] elements and ethical principles are applied to the practice of live donor transplantation."16 The donor advocate's primary obligation would be to "help the donor understand the process, procedure, and risks and benefits of live organ donation and to protect 
and promote the interests and well-being of the donor." There are clear limits to the influence of the donor advocate on the donor. The donor advocate does not have the right to prevent the donor from undergoing the donor surgery because, by law, this decision ultimately is made by the donor. However, the donor advocate may help the potential donor consider the risk of the donor surgery independent from the deliberations of the transplant team.

\section{Liver Biopsy}

The role of liver biopsy in the donor evaluation process varies greatly from center to center. Some centers perform liver biopsy on all potential donors, whereas others perform liver biopsy on potential donors based only on clinical findings that suggest some degree of concern regarding histological status of the liver, i.e., significant history of alcohol intake, BMI greater than $28 \mathrm{~kg} / \mathrm{m}^{2}$ (selected patients), elevated serum ferritin level, presence of steatosis on imaging studies, and so on. Liver biopsy can determine the presence of any underlying histological liver damage and determine the presence and extent of hepatic steatosis. Protocol liver biopsies in an otherwise suitable donor with normal liver function test results may discover minimal abnormalities (minimal portal inflammation, $<10 \%$ steatosis). A recent preliminary report of histological findings in potential donors subjected to protocol liver biopsy found that 73\% had an "abnormal liver biopsy." 18 Most abnormalities were nonspecific findings of unknown significance, e.g., granuloma or less than $10 \%$ steatosis. Whether potential donors with minimal histological abnormalities should be excluded from donation is unclear.

\section{Cost of Donor Evaluation}

Financial considerations of the donor evaluation and hepatectomy are important to consider because the process is complex and expensive. In general, all costs related to the donor are charged to the recipient's insurance carrier. Included in this fee are "failed donor evaluations," i.e., potential donors who undergo testing and are rejected for donation. We recently analyzed the total cost of donor evaluation and hepatectomy (including donor evaluation, donor hepatectomy, all medical care of the donor for 365 days after donation, and all failed donor evaluations). ${ }^{19} \mathrm{We}$ found that the cost of acquiring an organ from a live donor is almost identical (within 5\%) to that from a cadaveric donor. The distribution of donor costs was as follows: donor evaluation, 7\%; donor hepatectomy, 73\%; and follow-up care during the 365 days after donation, $20 \%$.

\section{Hepatitis B Core Antibody-Positive Donor}

Donors who are hepatitis B virus (HBV) surface antigen positive are excluded from donation. However, donors who are HBV core antibody and HBV surface antigen negative have been successfully used as living liver transplant donors. ${ }^{20-23}$ (Donors who are HBV core antibody positive and HBV surface antigen negative do not have active $\mathrm{HBV}$ infection, but previous exposure to HBV.) The consideration of HBV core antibodypositive donors is particularly important in regions of the world where $\mathrm{HBV}$ infection is endemic and a large number of LDLT procedures are performed. For example, in many parts of Asia, LDLT is the only option for liver transplantation, and more than $50 \%$ of the (blood donor) population may be $\mathrm{HBV}$ core antibody positive. $^{21}$

Special care must be used to ensure that the HBV core antibody-positive donor is able to yield a suitable graft. The presence of HBV core antibody indicates previous $\mathrm{HBV}$ infection, which can result in chronic liver damage. Therefore, liver biopsy is mandatory in the HBV core antibody-positive donor. The presence of hepatic fibrosis would preclude donor hepatectomy on the basis of graft suitability, as well as donor safety. The safety and success of LDLT in appropriately selected $\mathrm{HBV}$ core antibody-positive donors has been evaluated in several centers in Asia, where there are a large number of HBV core antibody-positive individuals. These studies have shown that the risk of hepatectomy in the HBV core antibody-positive donor is not greater than in other donors. ${ }^{21}$

Another concern related to the use of HBV core antibody-positive donors in LDLT is transmission of HBV from donor to recipient. In cadaveric transplantation, recipients of grafts from HBV core antibodypositive donors acquire posttransplantation $\mathrm{HBV}$ infection in up to $75 \%$ of cases. (Recipient acquisition of $\mathrm{HBV}$ infection from an HBV core antibody-positive donor likely is caused by reactivation of minute amounts of HBV in the donor organ, which occurs because of immunosuppression.) In LDLT, there are documented cases of HBV transmission from HBV core antibody-positive donors to living donor liver transplant recipients who have not been administered standard HBV prophylaxis. ${ }^{23}$ Administration of HBV prophylaxis with either hepatitis $B$ immunoglobulin ${ }^{23}$ or lamivudine ${ }^{22}$ to living donor liver transplant recipients of grafts from HBV core antibody-positive donors successfully prevents the acquisition of recipient HBV. Based on this experience, recipients of grafts from HBV core antibody-positive donors should be administered 
prophylaxis with either hepatitis B immunoglobulin, lamivudine, or both.

\section{Fulminant Liver Failure}

LDLT has been performed successfully in the setting of acute liver failure. ${ }^{24-29}$ However, the number of cases performed in the United States is very small. Based on UNOS statistics between July 2001 and June 2002, only $5.5 \%$ of 476 living donor liver transplant recipients (26 patients) underwent transplantation as UNOS status 1 , which represents only $0.5 \%$ of all liver transplantations (26 transplantations) performed in the United States. ${ }^{6}$ There are special concerns related to the donor evaluation in the setting of acute liver failure. Most important, the donor evaluation must be performed rapidly because of the severity of illness in the recipient and the rapid clinical evolution of acute liver failure. As a result, the potential donor's evaluation and decision to donate must occur during a very short interval; usually a few days. Some physicians have expressed concern that the expedited evaluation in the setting of acute liver failure potentially could preclude the potential donor from making a careful reasoned decision about donation..$^{30}$

In addition, the evaluation team must perform a careful, but expedited, evaluation of the donor. An expedited medical evaluation of the donor usually can be performed in rapid sequence. However, the psychosocial evaluation in some potential donors cannot be completed during the course of a few days. Because of concerns with an expedited donor evaluation, some centers have elected to exclude acute liver failure as an indication for LDLT. The role of LDLT in acute liver failure likely will remain limited because of the small number of patients with acute liver failure and complexities of the donor evaluation in this setting. However, in Asia, where cadaveric donor options may not exist, LDLT likely will continue to be a viable option for carefully selected patients.

\section{Ethnic Differences in Living Donor Liver Transplant Recipients}

A greater proportion of Hispanic patients undergo LDLT compared with cadaveric transplantation. In the United States, the proportion of Hispanic living donor liver transplant recipients $(16.8 \%)$ is $24 \%$ greater than Hispanic cadaveric transplant recipients (13.6\%). ${ }^{6} \mathrm{~A}$ report from Columbia-Presbyterian Hospital in New York evaluated the proportion of patients listed for cadaveric transplantation who had potential donors volunteer for evaluation. ${ }^{31}$ The investigators found that the proportion of Hispanic patients with potential donors was more than twofold greater than those without potential donors. At our center, the percentage of Hispanic transplant recipients undergoing LDLT $(35 \%)$ is more than twofold greater than Hispanics undergoing cadaveric transplantation (16\%).

The reason that Hispanics are more likely to undergo LDLT is unclear. One possibility is that a greater proportion of Hispanic patients may have favorable clinical characteristics for LDLT. In addition, Hispanics generally have larger families (with more potential donors) and may have a greater predisposition to donate. Based on these data, Hispanics may have greater access to LDLT than other ethnic groups, and a greater number of Hispanic may undergo the procedure. Because nonwhite cadaveric liver transplant recipients have decreased posttransplantation survival, differences in the ethnic makeup of living donor liver transplant recipients could impact on outcomes of the procedure. ${ }^{32,33}$

\section{References}

1. Trotter JF, Wachs M, Everson GT, Kam I. Adult-to-adult transplantation of the right hepatic lobe from a living donor. $\mathrm{N}$ Engl J Med 2002;346:1074-1082.

2. Trotter JF. Selection of donors and recipients for living donor liver transplantation. Liver Transpl 2000;6(suppl):S52-S58.

3. Schiano TD, Kim-Schluger L, Gondolesi G, Miller CM. Adult living donor liver transplantation: The hepatologist's perspective. Hepatology 2001;33:3-9.

4. Marcos A, Fisher RA, Ham JM, Olzinski AT, Shiffman ML, Sanyal AJ, et al. Selection and outcome of living donors for adult to adult right lobe transplantation. Transplantation 2000;69: 2410-2415.

5. Chen YS, Cheng YF, De Villa VH, Wang CC, Lin CC, Huang TL, et al. Evaluation of living liver donors. Transplantation 2003;75(suppl):S16-S19.

6. United Network for Organ Sharing. Annual Report of the Scientific Registry of Transplant Recipients and the Organ Procurement and Transplantation Network: Transplant data 2003. Available at: http://www.unos.org/latestdata/rptdatabase. Accessed April 15, 2003.

7. Trotter JF, Wachs M, Trouillot T, Steinberg T, Bak T, Everson GT, Kam I. Evaluation of 100 patients for living donor liver transplantation. Liver Transpl 2000;6:290-295.

8. Urata K, Kawasaki S, Matsunami H, Hashikura Y, Ikegami T, Ishizone $S$, et al. Calculation of child and adult standard liver volume for liver transplantation. Hepatology 1995;21:13171321.

9. Ben-Haim M, Emre S, Fishbein TM, Sheiner PA, Bodian CA, Kim-Schluger L, et al. Critical graft size in adult-to-adult living donor liver transplantation: Impact of the recipient's disease. Liver Transpl 2001;7:948-953.

10. Mokdad AH, Bowman BA, Ford ES, Bowman BA, Dietz WH, Vinicor F, Bales VS, Marks JS. Prevalence of obesity, diabetes, and obesity related health risk factors, 2001. JAMA 2003:289; 76-79.

11. Rinella ME, Alonso E, Rao S, Whitington P, Fryer J, Abecassis 
$\mathrm{M}$, et al. Body mass index as a predictor of hepatic steatosis in living liver donors. Liver Transpl 2001;7:409-414.

12. Ryan CK, Johnson LA, Germin BI, Marcos A. One hundred hepatic biopsies in the workup of living donors for right lobe liver transplantation. Liver Transpl 2002;8:1114-1122.

13. Allison DB, Saunders SE. Obesity in North America. Med Clin North Am 2000;84:305-332.

14. Kral JG, Schaffner F, Pierson RN, Wang J. Body fat topography as an independent predictor of fatty liver. Metabolism 1993;42: 548-551.

15. Ad Hoc UNOS Committee on Living Donor Liver Transplantation: Personal communication, July 2003.

16. Ascher N. Secretary's Advisory Committee on Organ Transplantation. Health and Human Services Administration 2002.

17. New York State Committee on Quality Improvement in Living Liver Donation. New York State Health Department 2002.

18. Tran T, Colquhoun S, Changsri C, Shackleton C, Peterson A, Geller $S$, et al. Living donor liver transplantation: The majority of donors have histologic abnormalities on liver biopsy [abstract]. Gastroenterology 2003;124:5.

19. Trotter JF, Mackenzie S, Wachs M, Bak T, Steinberg T, Polsky $\mathrm{P}$, et al. Comprehensive cost comparison of adult-adult right hepatic lobe living-donor liver transplantation with cadaveric transplantation. Transplantation 2003;75:473-476.

20. de Villa VH, Chen YS, Chen CL. Hepatitis B core antibodypositive grafts: Recipient's risk. Transplantation 2003;75(suppl): S49-S53.

21. Hwang S, Moon DB, Lee SG, Park KM, Kim KH, Ahn CS, et al. Safety of anti-hepatitis B core antibody-positive donors for living-donor liver transplantation. Transplantation 2003; 75(suppl):S45-S48.

22. Chen YS, Wang CC, de Villa VH, Wang SH, Cheng YF, Huang TL, et al. Prevention of de novo hepatitis B virus infection in living donor liver transplantation using hepatitis B core antibody positive donors. Clin Transplant 2002;16:405-409.

23. Uemoto S, Sugiyama K, Marusawa H, Inomata $Y$, Asonuma K, Egawa $\mathrm{H}$, et al. Transmission of hepatitis $\mathrm{B}$ virus from hepatitis
B core antibody-positive donors in living related liver transplants. Transplantation 1998;65:494-499.

24. Liu CL, Fan ST, Lo CM, Yong BH, Fung AS, Wong J. Rightlobe live donor liver transplantation improves survival of patients with acute liver failure. Br J Surg 2002;89:317-322.

25. Nishizaki T, Hiroshige S, Ikegami T, Uchiyama H, Hashimoto K, Soejima Y, Shimada M. Living-donor liver transplantation for fulminant hepatic failure in adult patients with a left-lobe graft. Surgery 2002;131(suppl):S182-S189.

26. Uemoto S, Inomata Y, Sakurai T, Egawa H, Fujita S, Kiuchi T, et al. Living donor liver transplantation for fulminant hepatic failure. Transplantation 2000;70:152-157.

27. Marcos A, Ham JM, Fisher RA, Olzinski AT, Shiffman ML, Sanyal AJ, et al. Emergency adult to adult living donor liver transplantation for fulminant hepatic failure. Transplantation 2000;69:2202-2205.

28. Miwa S, Hashikura Y, Mita A, Kubota T, Chisuwa H, Nakazawa $\mathrm{Y}$, et al. Living-related liver transplantation for patients with fulminant and subfulminant hepatic failure. Hepatology 1999; 30:1521-1526.

29. Lo CM, Fan ST, Liu CL, Wei WI, Chan JK, Lai CL, et al. Applicability of living donor liver transplantation to high-urgency patients. Transplantation 1999;67:73-77.

30. Abouna GJ. Emergency adult to adult living donor liver transplantation for fulminant hepatic failure-Is it justifiable? Transplantation 2001;71:1498-1500.

31. Rudow DL, Russo MW, Hafliger S, Emond JC, Brown RS Jr. Clinical and ethnic differences in candidates listed for liver transplantation with and without potential living donors. Liver Transpl 2003;9:254-259.

32. Charlton M, Seaberg E, Wiesner R, Everhart J, Zetterman R, Lake J, et al. Predictors of patient and graft survival following liver transplantation for hepatitis C. Hepatology 1998;28:823830.

33. Nair S, Eustace J, Thuluvath P. Effect of race on outcome of orthotopic liver transplantation: A cohort study. Lancet 2002; 359:287-293. 